
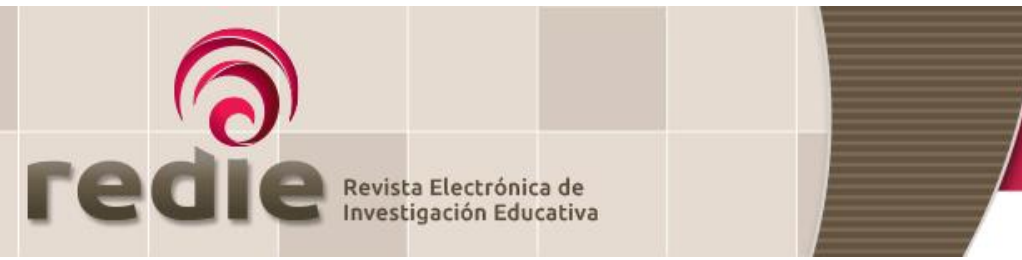

\title{
Influencia del conocimiento profundo del profesor sobre fracciones en el aprendizaje de alumnos de 40. grado
}

\section{Influence of In-Depth Teacher Knowledge of Fractions on Learning in $4^{\text {th }}$ Grade Students}

\author{
Palmenia de la Cruz Rodríguez Rojas (*) https://orcid.org/0000-0002-1703-0439 \\ Carlos Arturo Navarrete Rojas (*) https://orcid.org/0000-0002-2884-7882 \\ (*) Universidad de La Serena \\ (Recibido: 28 de febrero de 2018; Aceptado para su publicación: 13 de junio de 2018) \\ Cómo citar: Rodríguez, P. y Navarrete, C. A. (2020). Influencia del conocimiento profundo del profesor sobre fracciones en el \\ aprendizaje de alumnos de 4o. grado. Revista Electrónica de Investigación Educativa, 22, e10, 1-18. \\ https://doi.org/10.24320/redie.2020.22.e10.2285
}

\section{Resumen}

El objetivo de este estudio es establecer en qué medida el conocimiento profundo del profesor sobre fracciones y el conocimiento sobre la enseñanza de las mismas, ajustados por el nivel socioeconómico y el nivel de conocimientos en matemáticas que se alcanza en las escuelas (SIMCE), influye en el aprendizaje de los alumnos en cuanto a la conceptualización de las fracciones en 4o. grado. Con información de 378 alumnos de 4o. grado de 9 escuelas, así como de sus respectivos profesores de matemáticas, los resultados de los análisis de regresión muestran que el efecto Conocimiento Profundo del profesor es significativo en el aprendizaje por alumno ( $p=0.001)$, más allá de lo explicado por el puntaje SIMCE del colegio, que también es significativo $(p=0.02)$.

Palabras clave: Conocimiento del profesor, fracciones, enseñanza de las matemáticas.

\section{Abstract}

The objective of this study is to determine the extent to which teachers' in-depth knowledge of fractions and knowledge of how to teach them, adjusted for socioeconomic level and level of knowledge attained in mathematics in schools (SIMCE), influences student learning in conceptualizing fractions in 4 th grade. With information from 378 fourth-grade students in 9 schools and their mathematics teachers, the regression analysis results show that teachers' in-depth knowledge has a significant effect on student learning ( $p=0.001)$, beyond that explained by the school's SIMCE score, which is also significant $(p=0.02)$.

Keywords: Teacher knowledge, fractions, mathematics education.

\section{Introducción}

En las últimas tres décadas el conocimiento que los profesores requieren para impartir una enseñanza efectiva de los temas matemáticos específicos se ha convertido en un foco de creciente interés, tanto para investigadores como para autoridades educativas (Pino-Fan et al., 2018). En un período en que los países trabajan para elevar el nivel de conocimiento de matemáticas entre los estudiantes es importante que la formación inicial del profesor garantice que sus graduados dominan el conocimiento que requerirán para 
su práctica profesional. Cabe entonces preguntar cuáles son los conocimientos que requiere un profesor para realizar una enseñanza efectiva.

Shulman (1986) ha sido pionero en caracterizar de manera genérica el conocimiento para la enseñanza del contenido, él distingue entre conocimiento del contenido (cc), conocimiento pedagógico del contenido (CPC) y conocimiento curricular, definiendo al cc como "la cantidad y la organización del conocimiento per se en la mente de los maestros"; al CPC como "la forma particular del conocimiento que incorpora el aspecto del contenido que guarda más relación con la enseñanza", y que incluye saber cómo representar el contenido para que otros lo comprendan. Por su parte, el conocimiento curricular involucra saber cómo se organizan los temas, tanto dentro de un año escolar como a lo largo del tiempo.

Desde que Shulman (1986) presentara estas categorías, otros investigadores han descrito diversas conceptualizaciones acerca de lo que los profesores necesitan saber para enseñar matemáticas (Ball et al., 2008; Carrillo et al., 2013; Davis y Simmt, 2006; Hill et al., 2008; Ma, 1999; Rowland et al., 2005; Stylianides y Stylianides, 2014; Tchoshanov et al., 2017).

Ball et al. (2008) caracterizaron el conocimiento requerido para enseñar matemáticas y mediante el modelo conocimiento matemático para la enseñanza presentaron categorías asociadas al cc: conocimiento común del contenido, conocimiento especializado del contenido y conocimiento del horizonte matemático. Hill et al. (2008) contribuyendo a precisar este conocimiento, establecieron tres categorías del CPC: conocimiento de la enseñanza del contenido, conocimiento del currículo y conocimiento de los estudiantes y el contenido matemático.

Ma (1999), alineada con la conceptualización de Shulman (1986), mostró el conocimiento que ponen en juego en la tarea de enseñar profesores estadounidenses y chinos; en su trabajo se evidencian problemas propios de la enseñanza de la matemática. El principal hallazgo de Ma se relaciona con la noción de comprensión profunda de las matemáticas fundamentales (CPMF). Un profesor con CPMF posee un conocimiento profundo del currículo escolar, conceptual e interconectado que "va más allá de poder calcular en forma correcta y dar fundamentos para algoritmos de cálculo" (p. 9). La noción de CPMF se muestra habilitadora del conocimiento didáctico de las matemáticas.

La literatura da cuenta de la importancia del conocimiento del profesor sobre las matemáticas que enseña. No obstante, en el informe National Mathematics Advisory Panel [NMAP] (2008) se señala que es necesario investigar más sobre los tipos de conocimiento de los profesores que se relacionan con lo que los estudiantes aprenden. El NMAP (2008) analizó investigaciones en las que se utilizaron variables sustitutas para medir el conocimiento del profesor, tales como: certificados, cursos de matemáticas y/o grado. Sin embargo, estos estudios mostraron resultados inconsistentes, en general no confirman la importancia del conocimiento matemático de los profesores en relación con el aprendizaje de los estudiantes.

El NMAP (2008) señala que el único estudio que midió directamente el conocimiento matemático utilizado para enseñar y su incidencia en los logros de aprendizaje es el trabajo de Hill et al. (2005), quienes muestran que el conocimiento matemático para la enseñanza de los profesores se asocia de manera significativa con el aprendizaje en matemáticas de estudiantes de primer y tercer grado. Posteriormente se publicó el trabajo de Baumert et al. (2010), cuyos resultados revelaron un efecto positivo del CPC sobre el avance en el aprendizaje de estudiantes de 10o. grado en matemáticas. Cueto et al. (2017) encuentran una asociación positiva entre el CPC de los profesores y el rendimiento en matemáticas de estudiantes de 40. grado.

Tchoshanov (2011) parte de un supuesto similar al NMAP (2008) al señalar que no sólo importa la cantidad de cursos de matemáticas que tiene un profesor, sino saber qué tipo específico de conocimiento matemático posee un profesor efectivo. El objetivo de su estudio consistió en medir tres tipos cognitivos del conocimiento matemático de profesores y su asociación con el éxito de estudiantes de 60. a 80. grado. El principal hallazgo de su estudio fue que el conocimiento de los conceptos y las conexiones del profesor se asocia significativamente con el logro del estudiante. Posteriormente Tchoshanov et al. (2017) estudiaron la asociación entre tres tipos cognitivos del conocimiento matemático de los profesores y el desempeño de estudiantes de 5o. a 9o. grado. Los resultados sugieren que el conocimiento del contenido 
de los docentes juega un papel importante en el rendimiento de los estudiantes.

Las investigaciones existentes muestran que el conocimiento de los profesores sobre la matemática que enseñan se asocia de manera significativa y tiene un efecto positivo en el aprendizaje de los estudiantes. Sin embargo, en países en desarrollo faltan estudios sobre la relación entre el conocimiento de los profesores y el aprendizaje de los alumnos (Cueto et al., 2017) y más aún sobre temas matemáticos específicos. En este estudio se aborda la conceptualización de las fracciones, un tema complejo y de gran interés para la matemática educativa.

Estudiantes de todo el mundo tienen dificultades para aprender las fracciones, aun en países donde la mayoría de los estudiantes obtiene una comprensión conceptual aceptable, como Japón o China, las fracciones son consideradas un tema difícil (Fazio y Siegler, 2011). Las dificultades que enfrentan los alumnos para realizar operaciones básicas con fracciones conduce a errores comunes que aparecen en el aprendizaje del álgebra, que conforma una base fundamental en las matemáticas de formación superior (Siegler et al., 2012; Torbeyns et al., 2015). Hay investigaciones que muestran que los profesores presentan dificultades similares a las identificadas en los alumnos (Jakobsen et al., 2014; Ma, 2010; Pinto y Ribeiro, 2013). La falta de conocimiento matemático para la enseñanza puede afectar la forma en que los profesores critican los libros de texto, seleccionan el material para enseñar, estructuran sus cursos y conducen la instrucción (Crossman et al., 2005).

Este estudio tiene como propósito mostrar evidencias del efecto positivo que tiene el conocimiento del profesor sobre el aprendizaje del alumno en la conceptualización de las fracciones, considerando dos tipos de conocimientos del profesor, el "conocimiento profundo de las fracciones" y el "conocimiento sobre la enseñanza de las fracciones" (Rodríguez y Olfos, 2018).

Рara efectos de este trabajo, el constructo conocimiento profundo de las fracciones se define como un conocimiento conceptual e interconectado (Gallardo et al., 2008; Ma, 2010). En el campo de la cognición matemática existe cierto acuerdo en diferenciar dos tipos de conocimientos: el conceptual y el procedimental; el conocimiento conceptual se define como el conocimiento de los conceptos, mientras el procedimental es la capacidad de ejecutar procedimientos o pasos para resolver un problema (RittleJohnson y Schneider, 2014).

Varios investigadores señalan que el conocimiento conceptual de las fracciones explica el aprendizaje de matemáticas más avanzadas; por ejemplo, Byrnes y Wasik (1991) mostraron que el conocimiento conceptual de las fracciones explica el aprendizaje de la capacidad de realizar procedimientos de adición y multiplicación con fracciones. De manera análoga, los hallazgos de Charalambous y Pitta-Pantazi (2007) indican que el conocimiento conceptual predice el desempeño procedimental. Por su parte, Fuchs et al. (2013) señalan que el conocimiento conceptual tiende a tener un efecto mayor sobre el desarrollo del conocimiento procedimental y no a la inversa, y Rittle-Johnson et al. (2015) indican que existe cierto consenso en señalar que el conocimiento conceptual a menudo apoya y conduce al conocimiento procedimental.

Pero, ¿qué significa tener un conocimiento conceptual de las fracciones? Algunos investigadores proponen que el conocimiento conceptual de las fracciones incluye la comprensión de sus diferentes subconstructos, tales como: parte-todo, cociente, operador, razón y medida (Behr et al., 1993; Escolano y Gairín, 2005; Gallardo et al., 2008; Kieren, 1993). Fuchs et al. (2014), por su parte, señalan que en 4o. grado la comprensión del subconstructo parte-todo se representa típicamente utilizando un modelo de área y que la comprensión del subconstructo medida de las fracciones a menudo se representa con rectas numéricas. Los hallazgos de la investigación de Fuchs et al. (2013; 2014) sugieren que la enseñanza centrada en la fracción como medida sería clave para el aprendizaje de las fracciones.

Por otra parte, la literatura advierte que el conocimiento que requiere un profesor para enseñar matemáticas no se limita al conocimiento matemático. Existe un conocimiento complementario que se mantiene en discusión desde que Shulman (1986) identificara el CPC. Hill et al. (2008) identificaron un componente del CPC al que llamaron "conocimiento de los estudiantes y el contenido matemático", y lo 
definieron como "el conocimiento de cómo los estudiantes piensan, saben o aprenden un contenido en particular" (p. 375). Dicho componente del CPC incluye conocer las concepciones erróneas, dificultades y estrategias utilizadas por el alumno, por ejemplo: los errores o malentendidos que comúnmente surgen cuando los estudiantes aprenden a sumar fracciones.

Con base en la investigación de Hill et al. (2008), en este estudio el constructo "conocimiento sobre la enseñanza de las fracciones" se refiere al conocimiento que tiene el profesor respecto a las dificultades, errores comunes y estrategias utilizadas por los estudiantes. En cuanto a las dificultades y errores que se presentan en el uso de las fracciones, varios investigadores señalan que se deben tanto a la gran cantidad de subconstructos que tienen como a los conocimientos previos que tienen los alumnos. Por ejemplo, presentan dificultades con tareas asociadas al subconstructo de medida, como colocar fracciones en una recta numérica, determinar fracciones equivalentes, comparar y ordenar fracciones (Cortina et al., 2012; Fandiño, 2009; Godino, 2004; Hansen et al., 2015).

Por citar algunos ejemplos, al comparar fracciones Godino (2004) señala que los niños se equivocan al señalar que $1 / 2$ es menor que $1 / 3$, argumentando que 2 es menor que 3 . También se equivocan cuando señalan que la mitad de la fracción $1 / 6$ se designa frecuentemente por la fracción 1/3, argumentando que la mitad de 6 es 3; así, las propiedades de los números naturales pueden ser un obstáculo para el aprendizaje de las fracciones. Los niños no comprenden que cualquier número entero puede dividirse en cualquier número de partes iguales; por ejemplo, pueden presentar dificultades cuando se les pide que repartan 3 tabletas de chocolate entre 5 niños.

Charalambous y Pitta-Pantazi (2007) examinan asociaciones entre los diferentes subconstructos de las fracciones (parte-todo, razón, operador, cociente y medida) en estudiantes de 5o. y 6o. grado. El estudio mostró que los estudiantes tuvieron más éxito en tareas relacionadas con el subconstructo parte-todo y menos éxito en tareas correspondiente al subconstructo medida. Fandiño (2009) señala que los estudiantes presentan dificultades en el reconocimiento de esquemas de parte-todo continuo y discreto. Butto (2013) reporta que estudiantes de 6o. grado de primaria presentan dificultades con actividades relacionadas con el subconstructo parte-todo discreto y con el subconstructo de medida, por ejemplo, ubicar fracciones en la recta numérica y equivalencia de fracciones.

Atendiendo a lo hasta ahora expuesto, el objetivo de este estudio es establecer en qué medida el Conocimiento Profundo y el Conocimiento sobre la Enseñanza de las fracciones del profesor, ajustado por las variables nivel socioeconómico (NSE) y nivel académico que alcanzan las escuelas en matemáticas (SIMCE) influyen sobre el conocimiento que alcanzan los alumnos en la conceptualización de las fracciones en 4o. grado.

El estudio consideró las siguientes preguntas de investigación: ¿El NSE influye en el aprendizaje de los alumnos en la conceptualización de las fracciones? ¿El SIMCE influye en el aprendizaje de los alumnos en la conceptualización de las fracciones? ¿El conocimiento del profesor otorga un efecto adicional a lo que atribuye al SIMCE y al NSE?; así, este estudio expo-facto con prueba inicial y prueba final, utilizando la técnica de regresión lineal múltiple, explora el conocimiento del profesor como factor explicativo del avance o logro de aprendizaje del alumno en fracciones.

\section{Método}

En el estudio participaron 378 estudiantes de 4o. grado atendidos por 10 profesores de 9 establecimientos escolares (3 municipales y 6 subvencionados), proporcional a la cantidad de estos tipos de escuelas en la ciudad de La Serena (Chile), correspondiente al 10\% de la población de establecimientos. Los grupos se seleccionarion a través un muestreo proporcional, con participación voluntaria. Los datos fueron tomados durante el año escolar 2015 (ver tabla I). De los 10 profesores 7 son mujeres y 3 hombres y en promedio tienen 14 años de experiencia, con un mínimo de 2 años y un máximo de 34. Los docentes tienen el título de profesor (9 en Educación Básica y 1 en Matemáticas). 
Tabla I. Tipos de escuela, número de cursos y alumnos por escuela

\begin{tabular}{c|c|c|c}
\hline Escuelas & Tipo & Cursos & Alumnos \\
\hline E1 & P-Sub & 1 & 28 \\
E2 & P-Sub & 2 & 48 \\
E3 & P-Sub & 2 & 47 \\
E4 & P-Sub & 2 & 58 \\
E5 & P-Sub & 1 & 18 \\
E6 & Mun & 1 & 9 \\
E7 & Mun & 1 & 26 \\
E8 & P-Sub & 3 & 113 \\
E9 & Mun & 2 & 31 \\
\hline Total & 9 & 15 & 378 \\
\hline
\end{tabular}

\subsection{Instrumentos}

Prueba sobre las fracciones para los alumnos. La prueba para 4o. grado se conforma de 22 ítems de opción múltiple organizados de acuerdo a una matriz de especificaciones enmarcada en los contenidos curriculares (Ministerio de Educación [Mineduc], 2012) de la unidad de "Fracciones", según muestra la tabla II. La prueba se aplicó a los alumnos al inicio del año escolar como Prueba inicial y al final del año como Prueba final. La prueba mostró una confiabilidad de .77 según el coeficiente alfa de Cronbach.

Tabla II. Matriz de especificaciones Prueba para los Alumnos

\begin{tabular}{l|c}
\hline & ítems \\
\hline Conocimientos previos & 6 \\
Comprensión de las fracciones & 9 \\
Adición y sustracción de fracciones de igual denominador en contexto & 3 \\
Identificación y representación de fracciones propias y números mixtos hasta 5 & 4 \\
\hline
\end{tabular}

Prueba sobre el Conocimiento Profundo y sobre la Enseñanza de las fracciones para el profesor. Las pruebas se conformaron por 10 ítems referidos al conocimiento profundo sobre las fracciones y 14 ítems sobre la enseñanza de las fracciones. Los antecedentes de las pruebas se encuentran en Rodríguez y Olfos (2018).

\subsection{Variables contextuales}

SIMCE. Corresponde al puntaje promedio desde el 2011 al 2015 obtenido por la escuela en las pruebas de matemáticas anuales SIMCE (Sistema de medición de la calidad de la educación) realizadas en el país, que evalúan el logro de aprendizaje en la asignatura de matemáticas, abarcando una muestra representativa de los contenidos curriculares de 30. y 40. grado (Mineduc, 2012). Los puntajes de la prueba SIMCE se encuentran disponibles en http://www.agenciaeducacion.cl

NSE. Corresponde al nivel socioeconómico medio de las familias de los estudiantes que anualmente rinden las pruebas SIMCE en el país. En el grupo NSE Medio Bajo la mayoría de los apoderados ha declarado tener hasta 10 años de escolaridad y un ingreso del hogar de hasta $\$ 340,000$. En el grupo NSE Alto la mayoría de los apoderados ha declarado tener entre 13 y 15 años de escolaridad y un ingreso entre \$550,001 y 1,250,000. Los puntajes NSE son públicos y están disponibles en http://www.agenciaeducacion.cl

\subsection{Procedimiento}

Para la administración de las pruebas a los alumnos se solicitó autorización a los profesores, directores y apoderados. Las pruebas fueron administradas por un asistente del proyecto en las primeras horas de las jornadas de clases habituales de los alumnos, dando 60 minutos de tiempo. La prueba a los profesores fue administrada en conjunto a todos en una sala, dando 30 minutos de tiempo para responder a las preguntas de la prueba "Conocimiento profundo de las fracciones", luego de un descanso de 10 minutos, se dieron 
40 minutos para responder a la prueba "Conocimiento sobre la enseñanza de las fracciones". Se proveyó a los docentes un estímulo económico por su participación en el proyecto.

Las siguientes variables se consideran independientes: SIMCE, NSE, Conocimiento Profundo de las fracciones y Conocimiento sobre la Enseñanza de las fracciones. Los resultados del aprendizaje de los alumnos se evaluaron por medio de la variable dependiente "Avance" que se obtiene al calcular la diferencia entre el puntaje final y el puntaje inicial por alumno. La variable "Avance" considera a cada alumno como su propio referente, pero presupone un potencial de avance similar entre todos los alumnos. Para evaluar la normalidad se utilizó la prueba Kolmogorov-Smirnov y la homocedasticidad se analizó por medio de la prueba de Bartlett. La comparación del puntaje promedio SIMCE de la muestra con el promedio de los colegios de La Serena desde el 2011 al 2015 se realizó mediante una prueba $t$, los valores se presentan como medias y desviaciones estándar (DE). Las correlaciones que se presentan son correlaciones de Pearson. El ajuste por SIMCE y NSE se hizo a través de un modelo de regresión lineal múltiple.

\section{Resultados}

Dado que el interés de este estudio era conocer el valor predictivo de las variables "Conocimiento Profundo" y "Conocimiento sobre la Enseñanza" de las fracciones del profesor, ajustado por el nSE y el SIMCE en relación con la variable "Avance", se comprobó que no hay evidencia de falta de normalidad y no hay evidencia de heterocedasticidad para la variable Avance por Conocimiento Profundo $(p=0.13)$ y por Conocimiento sobre la Enseñanza $(p=0.29)$.

El puntaje promedio SIMCE de los colegios de La Serena desde el año 2011 al 2015 es de 260 (DE 24.5) y en los colegios de la muestra es de 263.15 (DE 23.95). El puntaje promedio SIMCE de la muestra no es significativamente distinto del promedio SIMCE de los colegios de La Serena $(p=0.68)$, como muestra la figura 1.

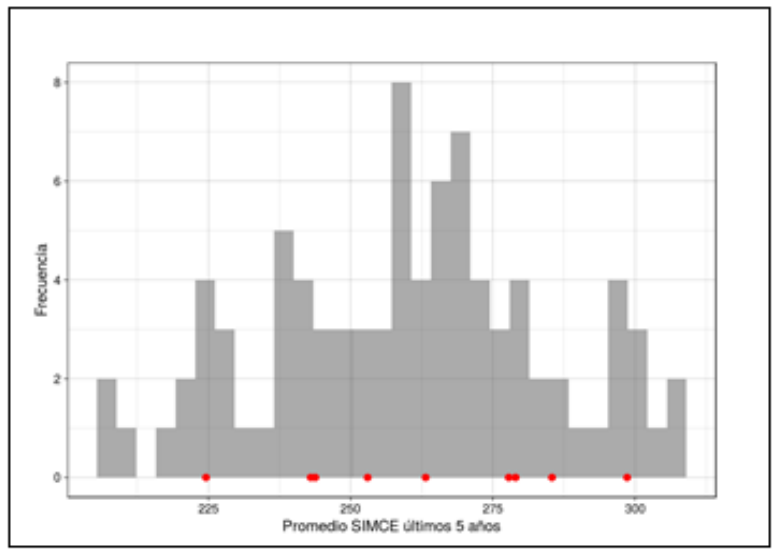

Figura 1. Distribución poblacional SIMCE de colegios de La Serena

La correlación entre el SIMCE con la Prueba final y la Prueba inicial por alumno es estadísticamente significativa ( $r=0.52, p=0.0001$ y $r=0.37, p=0.0001$, respectivamente), como evidencia la figura 2 . 


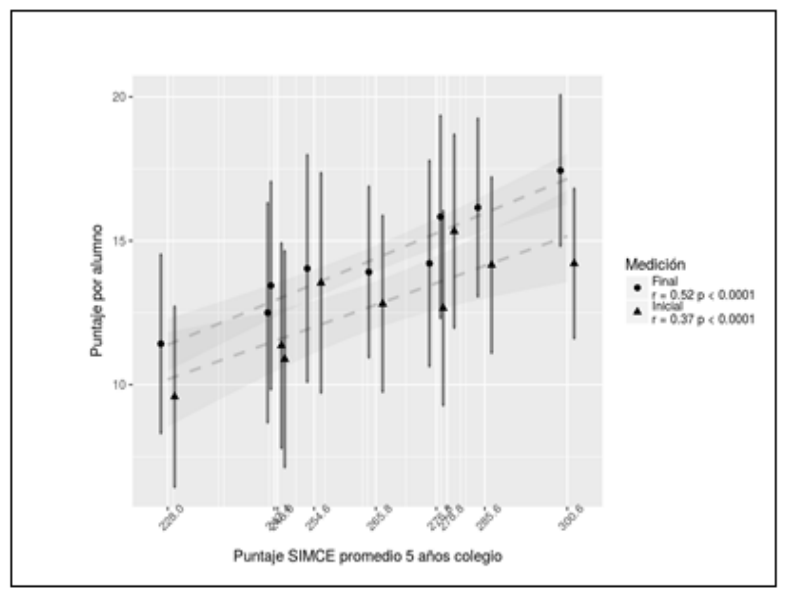

Figura 2. Correlación entre el SIMCE y el puntaje por alumno

El SIMCE se correlaciona de forma significativa con el NSE $(r=0.79, p=0.0001)$. El puntaje promedio SIMCE para el grupo NSE Alto es 286.33 (DE 13.1) y para el grupo NSE Medio Bajo es 243.05 (DE 10.99). El puntaje promedio SIMCE es significativamente mayor para el grupo del NSE Alto $(p=0.0001)$, ver figura 3.

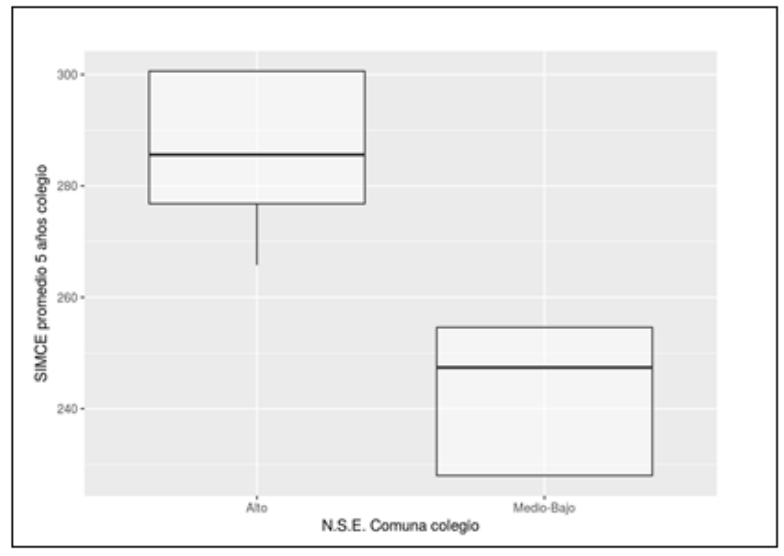

Figura 3. Diferencia SIMCE entre el grupo NSE Alto y NSE Medio Bajo

Como se observa en la figura 4, la asociación entre la Prueba inicial y la Prueba final es estadísticamente significativa ( $r=0.65, p=0.0001)$, mientras el NSE de la comuna por colegio es significativamente diferente $(p=0.0001)$ 


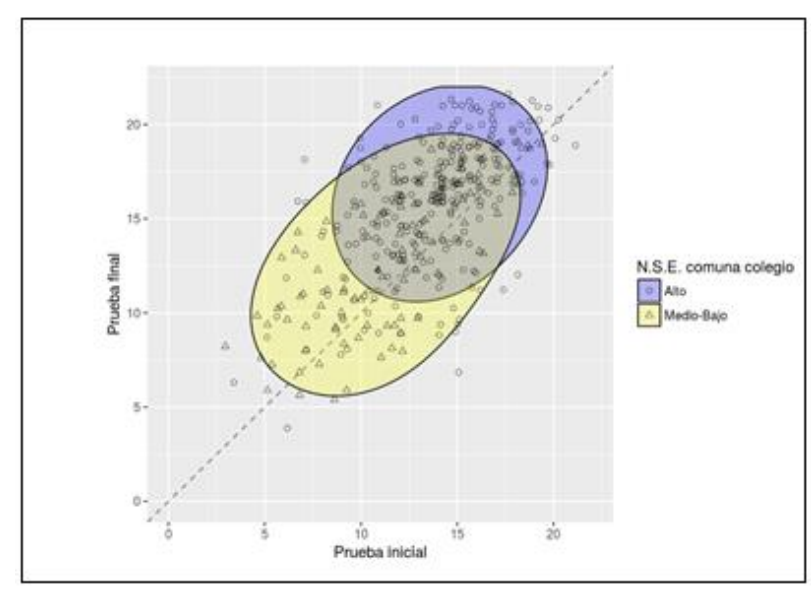

Figura 4. Relación entre el puntaje de las pruebas inicial y final

El puntaje del Avance por alumno se correlaciona de forma significativa con el puntaje SIMCE promedio del colegio al que pertenece $(r=0.21, p=0.0001)$, como ilustra la figura 5 .

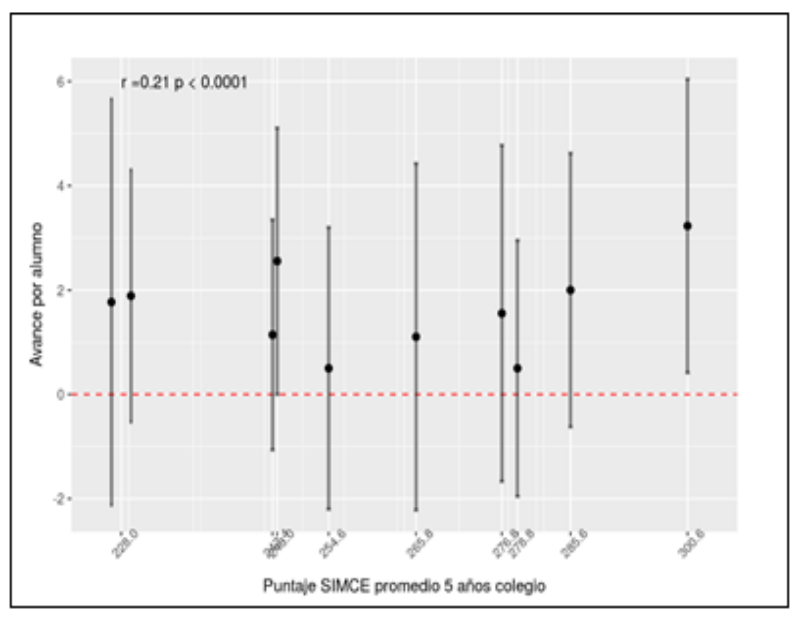

Figura 5. Relación entre el Avance y el SIMCE

El puntaje promedio del Avance por alumno para el grupo NSE Alto es 2.2 (DE 3.05) y para el grupo NSE Medio Bajo es 1.3 (DE 2.72). El puntaje promedio del Avance por alumno es significativamente mayor para el grupo del NSE Alto $(p=0.01)$, como se ve en la figura 6 . 


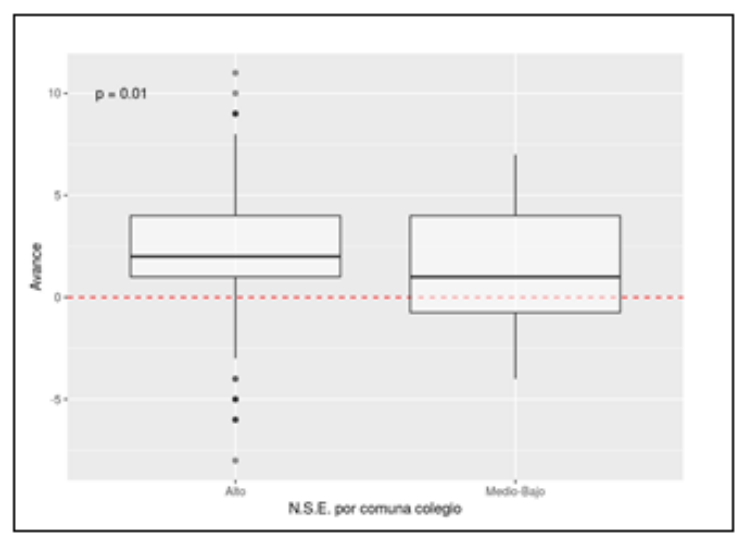

Figura 6. Diferencia del Avance entre el grupo NSE alto y NSE Medio Bajo

La correlación entre el Conocimiento Profundo y el puntaje promedio del Avance por alumno es estadísticamente significativa ( $r=0.25, p=0.0001)$, como se muestra en la figura 7 .

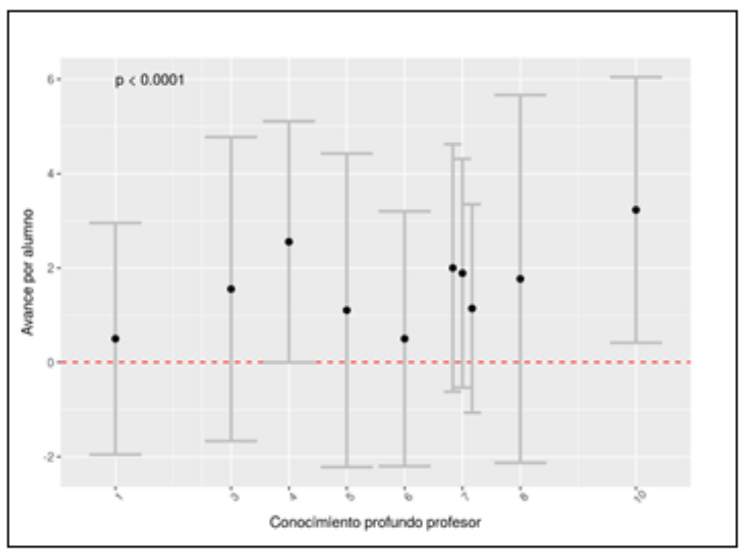

Figura 7. Relación entre Conocimiento profundo y Avance por alumno

La correlación entre el Conocimiento para la Enseñanza del profesor y el puntaje promedio del Avance por alumno es estadísticamente significativa $(r=0.17, p=0.001)$ (ver figura 8$)$.

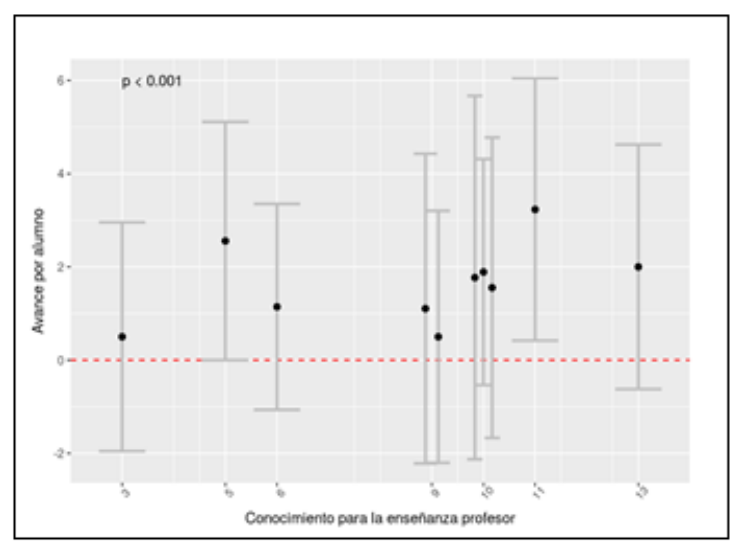

Figura 8. Relación entre Conocimiento para la enseñanza y Avance por alumno 
Los resultados de los análisis de regresión muestran que el efecto Conocimiento Profundo del profesor es significativo en el Avance por alumno ( $p=0.001)$, más allá de lo explicado por el puntaje sIMCE del colegio, que también es significativo $(p=0.02)$. En cambio, el efecto de la dimensión Conocimiento para la Enseñanza no alcanza significación estadística si se ajusta por SIMCE $(p=0.09)$. Si en conjunto se incluyen SIMCE, Conocimiento Profundo y Conocimiento para la Enseñanza, lo más relevante parece ser el Conocimiento Profundo del profesor $(p=0.01)$ junto con el SIMCE $(p=0.03)$.

Los resultados del análisis de las preguntas de las pruebas administradas a alumnos y profesores son los siguientes:

Prueba sobre Fracciones. Las preguntas que resultaron más fáciles de responder correctamente por los alumnos (> 80\%), fueron las relativas a la categoría Conocimientos previos, específicamente las de partetodo continuo y comparación de fracciones de un mismo todo con igual denominador. Las preguntas que resultaron más difíciles (20\% a 50\%) fueron las relativas a la categoría comprensión de las fracciones, en específico las de mayor dificultad fueron las relativas a ubicar fracciones en la recta numérica (medida), comparar fracciones con distinto denominador (medida) y las de parte-todo discreto. A continuación se presentan cuatro ejemplos de preguntas.

La pregunta 5 de la prueba sobre fracciones (figura 9) relativa al subconstructo parte-todo continuo, la responde correctamente el $90 \%$ del total de los alumnos de 40. grado.

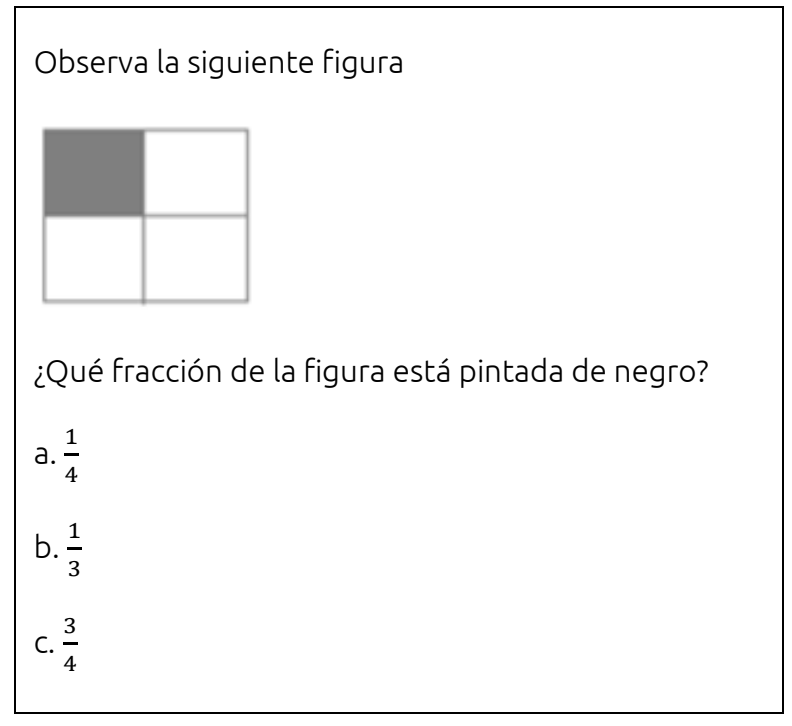

Figura 9. Prueba sobre fracciones subconstructo parte-todo continuo

La pregunta 6 de la prueba sobre fracciones (figura 10) relativa a comparar fracciones con igual denominador, la responde correctamente el $84 \%$ del total de los alumnos de 40 . grado.

Juan reparte un chocolate, él comió $\frac{2}{3}$ y su hermano comió $\frac{1}{3}$ del chocolate, ¿qué frase es correcta?

a. Juan comió tanto chocolate como su hermano.

b. Juan comió más chocolate que lo que comió su hermano.

c. Juan comió tres veces más chocolate que lo que comió su hermano.

Figura 10. Prueba sobre fracciones con igual denominador 
La pregunta 7 de la prueba sobre fracciones (figura 11) relativa al subconstructo de medida, la responde correctamente el 35\% del total de los alumnos de 4o. grado.

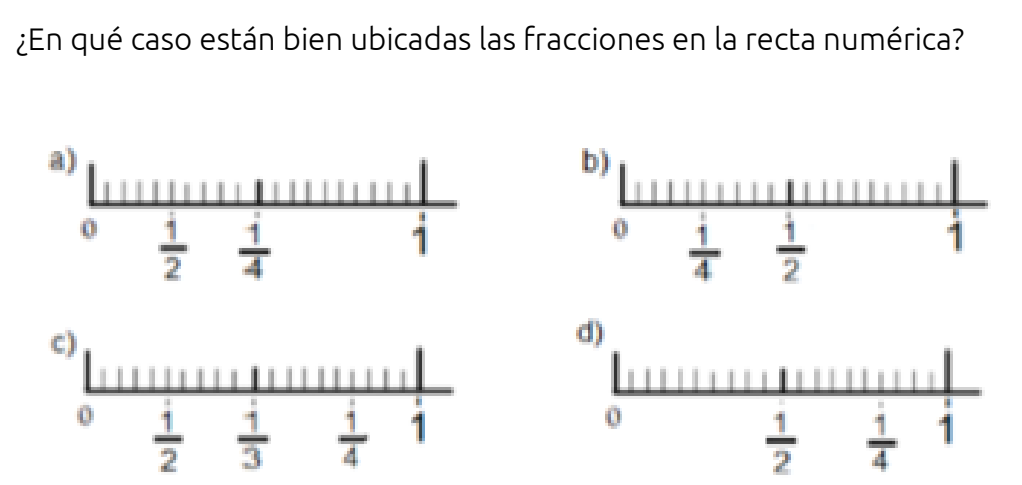

Figura 11. Prueba sobre fracciones subconstructo medida

La pregunta 8 de la prueba sobre fracciones (figura 12) relativa al subconstructo operador, la responde correctamente el $28 \%$ del total de los alumnos de 40 . grado.

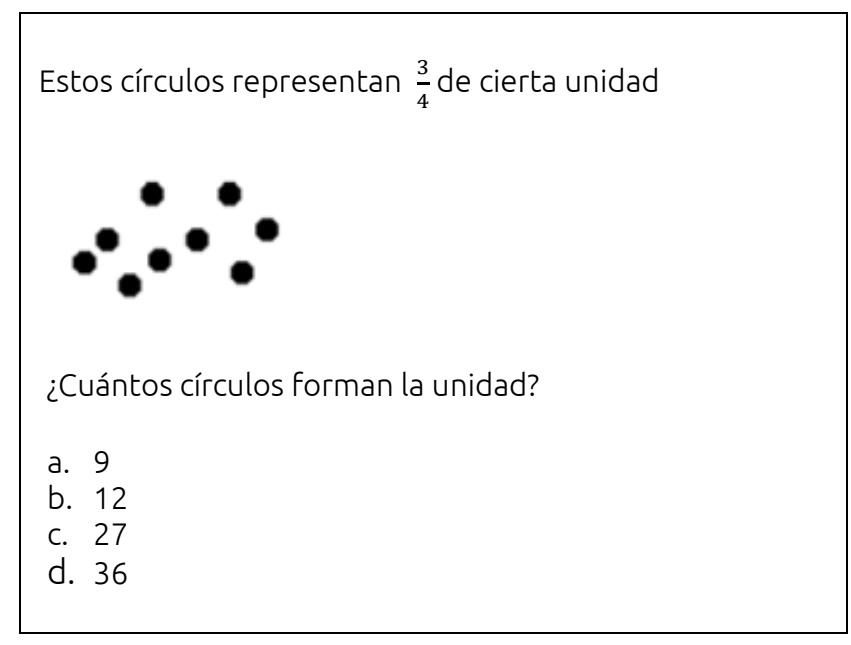

Figura 12. Prueba sobre subconstructo operador

Prueba sobre el Conocimiento Profundo. Las preguntas que resultaron más fáciles de responder correctamente por los profesores (> $80 \%$ ) fueron las relativas al subconstructo parte-todo continuo. Las preguntas que resultaron más difíciles (20\% a $50 \%$ ) fueron las relativas a indicar números fraccionarios entre dos números, ubicar fracciones en la recta numérica y calcular la fracción de un número.

La pregunta 5 de la prueba sobre Conocimiento Profundo (figura 13) relativa a indicar números fraccionarios entre dos números (subconstructo de medida), la responde correctamente el $40 \%$ del total de los profesores de primaria. 


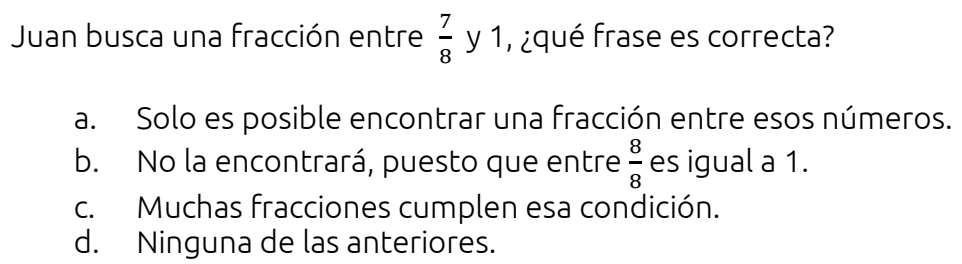

a. Solo es posible encontrar una fracción entre esos números.

b. No la encontrará, puesto que entre $\frac{8}{8}$ es igual a 1 .

c. Muchas fracciones cumplen esa condición.

d. Ninguna de las anteriores.

Fuente: Modificado de Rodríguez y Olfos (2018)

Figura 13. Prueba sobre el Conocimiento Profundo subconstructo medida

La pregunta 4 de la prueba sobre Conocimiento Profundo (figura 14) relativa a calcular la fracción de un número (subconstructo operador), la responde correctamente el 60\% del total de los profesores de primaria.

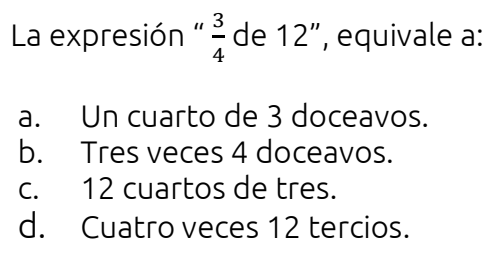

Figura 14. Prueba sobre Conocimiento Profundo subconstructo operador

Prueba sobre la Enseñanza de las fracciones. Las preguntas que resultaron más fáciles de responder correctamente (70\% a $90 \%$ ) por los profesores fueron las relativas a identificar dificultades comunes de los estudiantes, seguidas por las de conocer estrategias utilizadas por los alumnos. Las preguntas que resultaron más difíciles (30\% a 50\%) fueron las relativas al conocimiento de errores comunes de los alumnos.

La pregunta 14 de la prueba sobre la Enseñanza de las fracciones (figura 15) relativa a la categoría identificar dificultades comunes de los estudiantes, la responde correctamente el $90 \%$ del total de los profesores de primaria. 
Aquí hay dos segmentos de recta numérica de 2 unidades de longitud cada una. En cada segmento las distancias entre las marcas son iguales. La profesora solicitó escribir la fracción representada por la letra B. Algunos alumnos escribieron $\frac{3}{4}$.

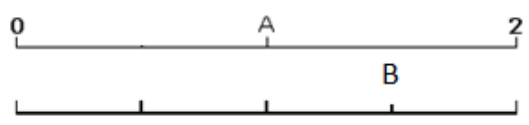

Ello puede deberse principalmente a que:
a. $\frac{3}{4}$ de 2 es $\frac{3}{2}$.
b. No me parece clara la pregunta.
c. La ubicación de A corresponde a 1.
d. B está ubicado en las tres cuartas partes del segmento de recta dibujado.

Fuente: Modificado de Rodríguez y Olfos (2018).

Figura 15. Prueba sobre la enseñanza de las fracciones: categoría identificar dificultades comunes

La pregunta 7 de la prueba sobre la Enseñanza de las fracciones (figura 16) relativa a la categoría conocimiento de errores comunes de los estudiantes, la responde correctamente el $30 \%$ del total de los profesores de primaria.

Fernanda señala que las siguientes fracciones $\frac{2}{5}$ y $\frac{8}{11}$ son iguales. ¿Qué pudo llevar a Fernanda a tal conclusión?

a. Fernanda se equivocó en encontrar los denominadores comunes.

b. Fernanda está agregando 6 , tanto al numerador como al denominador de $\frac{2}{5}$, y ella ve que eso es igual a $\frac{8}{11}$.

c. Pudo ser cualquiera de las dos ideas anteriores.

d. Ninguna de esas ideas se relaciona con la conclusión de Fernanda.

Figura 16. Prueba sobre la enseñanza de las fracciones: categoría conocimiento de errores comunes

\section{Discusión y conclusiones}

El presente estudio se enfocó en establecer en qué medida el Conocimiento Profundo y el Conocimiento sobre la Enseñanza de las fracciones del profesor, ajustados por las variables nivel socioeconómico (NSE) y nivel académico que alcanzan las escuelas en matemáticas (SIMCE), influyen sobre el conocimiento que alcanzan los alumnos en la conceptualización de las fracciones en 4o. grado. Se explora el conocimiento del profesor como factor explicativo del aprendizaje del alumno.

Los resultados de este estudio muestran que el NSE se correlaciona fuertemente y de forma significativa con el SIMCE $(r=0.79, p=0.0001)$. En Chile, los alumnos de escuelas que tienen un mayor NSE obtienen puntajes SIMCE significativamente más altos que los alumnos de escuelas que tienen un menor NSE (Mineduc, 2017; OCDE, 2018). Al comparar directamente las puntuaciones de las pruebas, los resultados se asocian con el NSE y el SIMCE. Tanto en Chile como en otros países en desarrollo, los alumnos con bajo NSE difícilmente puedan alcanzar rendimientos comparables con los alumnos de mejor NSE (OCDE, 2016). Por lo 
tanto, para evaluar los resultados del aprendizaje de los alumnos se utilizaron las puntuaciones de Avance de aprendizaje por alumno, ya que son estimaciones más justas del crecimiento académico de los estudiantes (Mullens et al., 1996).

En este estudio el puntaje SIMCE promedio se asocia significativamente con el puntaje promedio del Avance por alumno ( $r=0.21, p=0.0001)$ (ver figura 5$)$ y el puntaje promedio del Avance por alumno es significativamente mayor para el grupo del NSE alto $(p=0.01)$ (ver figura 6$)$. Sin embargo, los resultados de esta investigación muestran que si se compara al alumno en términos de Avance de aprendizaje, resulta que tanto los alumnos de NSE alto como los del NSE bajo aprenden y ese aprendizaje se asocia significativamente con el conocimiento profundo del profesor ( $r=0.25, p=0.0001$ ) (ver figura 7 ); de hecho, esa asociación va más allá de lo explicado por el SIMCE y el NSE. Aun ajustado por NSE y SIMCE, el conocimiento profundo del profesor marcó una diferencia $(p=0.01)$.

Los hallazgos de este estudio están en concordancia con las investigaciones que señalan que el conocimiento matemático para la enseñanza de los profesores se asocia significativamente e influye en el aprendizaje de los estudiantes. Por ejemplo, Harbison y Hanushek (1992) encuentran un efecto positivo entre el conocimiento matemático del profesor y el rendimiento de sus estudiantes en 4o. grado; Hill et al. (2005) muestran que el conocimiento matemático para la enseñanza de los profesores se relaciona significativamente con el logro en matemáticas de los estudiantes de primer y tercer grado; Tchoshanov (2011) encuentra que el conocimiento matemático de los conceptos y las conexiones del profesor se asocian significativamente con el logro del estudiante; mientras que Tchoshanov et al. (2017) encuentran que la puntuación total de la prueba de conocimiento del contenido de los profesores se correlaciona de forma significativa con el rendimiento de los estudiantes.

Los resultados de los análisis de la prueba sobre Fracciones muestran que las preguntas que resultaron más difíciles de responder correctamente por los alumnos fueron las relacionadas con el subconstructo de medida, específicamente ubicar fracciones en la recta numérica, comparar fracciones con distinto denominador y las relacionadas con el subconstructo parte-todo discreto. Lo que concuerda con lo que reporta la literatura, los estudiantes presentan dificultades al colocar fracciones en una recta numérica, al comparar fracciones y con actividades que requerían poner en práctica ideas matemáticas de fraccionamiento en cantidad discreta (Butto, 2013; Cortina et al., 2012; Charalambous y Pitta-Pantazi, 2007; Fandiño, 2009; Godino, 2004; Hansen et al., 2015).

Los resultados del análisis de la prueba sobre el conocimiento profundo de las fracciones muestran que las preguntas que resultaron más difíciles de responder correctamente por los profesores fueron las relacionadas con el subconstructo de medida (indicar números fraccionarios entre dos números, ubicar fracciones en la recta numérica) y las relacionadas con el subconstructo operador (calcular la fracción de un número). Estos resultados muestran que los profesores presentan dificultades similares a las identificadas en los alumnos, lo que coincide con lo reportado por Jakobsen et al. (2014), Ma (2010) y Pinto y Ribeiro (2013).

Los resultados de los análisis de la prueba sobre la enseñanza de las fracciones muestran que los profesores no tienen dificultad en reconocer las estrategias y dificultades comunes que tienen los estudiantes, pero sí en dimensionar la naturaleza del error. Los resultados sugieren que los profesores no conocen el porqué de los errores comunes que cometen los estudiantes.

Así, las investigaciones existentes sobre el conocimiento del profesor, medido por pruebas, y su relación con el rendimiento de los estudiantes muestran una tendencia prometedora (Tchoshanov, 2011). Como indica el NMAP (2008) al señalar que los resultados de pruebas administradas a los profesores constituirían una mejor medida de sus conocimientos matemáticos para la enseñanza que los certificados de títulos y grados, ya que una comprensión más detallada del conocimiento requerido para enseñar matemática sólo puede emerger al enfocarse en temas matemáticos específicos (Pino-Fan et al., 2018). De esta forma, la presente investigación constituye un aporte a la investigación sobre la relación entre el conocimiento del alumno y el conocimiento de los profesores. 
La situación socioeconómica de la familia de los estudiantes es un factor que influye en el rendimiento escolar (Chaparro et al., 2016; Murillo y Román, 2008; OCDE, 2016; Tuñón y Poy, 2016). Sin embargo, en este estudio se muestra que, independientemente del NSE, si se capacita al profesor de manera que tenga un conocimiento profundo sobre las fracciones se puede obtener un mejor avance por parte del alumno; es decir, preparar al profesor para que tenga un conocimiento conceptual e interconectado acerca de las fracciones (Rodríguez y Olfos, 2018) permitiría obtener el mejor avance de aprendizaje posible dadas las condiciones basales de los estudiantes. Se sugiere que en trabajos posteriores se consideren otras muestras y poblaciones de profesores para continuar la investigacion sobre cómo los diferentes tipos de conocimientos del docente se relacionan con el aprendizaje del estudiante.

\section{Referencias}

Ball, D., Thames, M. y Phelps, G. (2008). Content knowledge for teaching: what makes it special? Journal of Teacher Education, 59(5), 389-407. https://doi.org/10.1177/0022487108324554

Baumert, J., Kunter, M., Blum, W., Brunner, M., Voss, T. , Jordan A., Klusmann, U., Krauss, S., Neubrand, M. y Tsai, Y.-M. (2010). Teachers' mathematical knowledge, cognitive activation in the classroom, and student progress. American Educational Research Journal, 47(1), 133-180.

https://doi.org/10.3102/0002831209345157

Behr, M., Harel, G., Post, T. y Lesh, R. (1993). Rational numbers: toward a semantic analysis-emphasis on the operator construct. En T. Carpenter, E. Fennema y T. Romberg (Eds.), Rational numbers: an integration of research (pp. 13-47). Lawrence Erlbaum.

Butto, Z. C. (2013). El aprendizaje de fracciones en educación primaria: una propuesta de enseñanza en dos ambientes. Revista Horizontes Pedagogicos, 15(1), 33-45.

Byrnes, J. P. y Wasik, B. A. (1991). Role of conceptual knowledge in mathematical procedural learning. Developmental Psychology, 27(5), 777. https://doi.org/10.1037/0012-1649.27.5.777

Carrillo, J., Climent, N., Contreras, L. y Muñoz-Catalán, M. (2013). Determining specialized knowledge for mathematics teaching. Actas, VIII Congress of the European Society for Research in Mathematics Education [CERME] (pp. 2985-2994). Turquía: Middle East Technical University.

Chaparro, A., González, C. y Caso, J. (2016). Familia y rendimiento académico: configuración de perfiles estudiantiles en secundaria. Revista Electrónica de Investigación Educativa, 18(1), 53-68.

http://redie.uabc.mx/redie/article/view/774

Charalambous, C. Y. y Pitta-Pantazi, D. (2007). Drawing on a theoretical model to study students' understandings of fractions. Educational Studies in Mathematics, 64(3), 293-316.

https://doi.org/10.1007/s10649-006-9036-2

Cortina, J., Cardoso, E. y Zúñiga, C. (2012). El significado cuantitativo que tienen las fracciones para estudiantes mexicanos de 6o. de primaria. Revista Electrónica de Investigación Educativa, 14(1), 70-85.

https://redie.uabc.mx/redie/article/view/297/460

Cueto, S., León, J., Sorto, M. A. y Miranda, A. (2017). Teachers' pedagogical content knowledge and mathematics achievement of students in Peru. Educational Studies in Mathematics, 94(3), 329-345.

Davis, B. y Simmt, E. (2006). Mathematics-for-teaching: an ongoing investigation of the mathematics that teachers (need to) know. Educational Studies in Mathematics, 61(3), 293-319.

https://doi.org/10.1007/s10649-006-2372-4 
Escolano, R. y Gairín, J. M. (2005). Modelos de medida para la enseñanza de números racionales en educación primaria. UNIÓN Revista Latinoamericana de Educación Matemática, 1, 17-35.

Fandiño, M. I. (2009). Las fracciones. Aspectos conceptuales y didácticos. Magisterio.

Fazio, L. y Siegler, R. (2011). Enseñanza de las fracciones. UNESCO.

https://unesdoc.unesco.org/ark:/48223/pf0000212781 spa

Fuchs, L. S., Schumacher, R. F., Long, J., Namkung, J., Hamlett, C. L., Cirino, P. T., Jordan, N. C., Siegler, R., Gersten, R. y Changas, P. (2013). Improving at-risk learners' understanding of fractions. Journal of Educational Psychology, 105(3), 683-700. https://doi.org/10.1037/a0032446

Fuchs, L. S., Schumacher, R. F., Sterba, S. K., Long, J., Namkung, J., Malone, A., Hamlett, C. L., Jordan, N. C., Gersten, R., Siegler, R. S. y Changas, P. (2014) (2014). Does working memory moderate the effects of fraction intervention? An aptitude-treatment interaction. Journal of Educational Psychology, 106(2), 499514. https://doi.org/10.1037/a0034341

Gallardo, J., González, J. y Quispe, W. (2008). Interpretando la comprensión matemática en escenarios básicos de valoración. Un estudio sobre las interferencias en el uso de los significados de la fracción. Revista Latinoamericana de Investigación en Matemática Educativa, 11(3), 355-382.

Godino, J. (2004). Didáctica de las matemáticas para maestros. Proyecto Edumat-Maestros. http://www.ugr.es/ jgodino/edumat-maestros/manual/9 didactica maestros.pdf

Grossman, P., Wilson, S. y Shulman, L. (2005). Profesores de sustancia: el conocimiento de la materia para la enseñanza. Profesorado. Revista de Currículum y Formación del Profesorado, 9(2), 1-24

Hansen, N., Jordan, N. C. y Rodrigues, J. (2015). Identifying learning difficulties with fractions: a longitudinal study of student growth from third through sixth grade. Contemporary Educational Psychology, 50, 45-59 https://doi.org/10.1016/i.cedpsych.2015.11.002

Harbison, R. W. y Hanushek, E. A. (1992). Educational performance for the poor: lessons from rural northeast Brazil. Oxford University Press.

Hill, H., Ball, D. y Schilling, S. (2008). Unpacking pedagogical content knowledge: conceptualizing and measuring teachers' topic-specific knowledge of students. Journal for Research in Mathematics Education, 39(4), 372-400.

Hill, H., Rowan, B., y Ball, D. (2005). Effects of teachers' mathematical knowledge for teaching on student achievement. American Educational Research Journal, 42(2), 371-406.

https://doi.org/10.3102/00028312042002371

Jakobsen, A., Ribeiro, C. M. y Mellone, M. (2014). Norwegian prospective teachers' MKT when interpreting pupils' productions on a fraction task. Nordic Studies in Mathematics Education, 19(3-4), 135-150.

Kieren, T. (1993). Rational and fractional numbers: from quotient fields to recursive understanding. En T. Carpenter, E. Fennema y T. Romberg (Eds.), Rational numbers: an integration of research (pp. 49-84). Lawrence Erlbaum.

Ma, L. (1999). Knowing and teaching elementary mathematics: teachers' understanding of fundamental mathematics in China and the United States. Lawrence Erlbaum.

Ma, L. (2010). Conocimiento y enseñanza de las matemáticas elementales. La comprensión de las matemáticas fundamentales que tienen los profesores en China y los EE.UU. Academia Chilena de Ciencias. 
Ministerio de Educacion. (2012) Matemática. Programa de Estudio Cuarto Año Básico. Autor. http://www.curriculumenlineamineduc.cl/605/articles-18979 programa.pdf

Ministerio de Educacion. (2017). Revisión de las políticas educativas en Chile desde 2004 a 2016. Reporte Nacional de Chile. Autor.

https://centroestudios.mineduc.cl/wp-content/uploads/sites/100/2017/06/CBR Mineduc-WEB.pdf

Mullens, J., Murnane, R. y Willett, J. (1996). The contribution of training and subject matter knowledge to teaching effectiveness: a multilevel analysis of longitudinal evidence from Belize. Comparative Education Review, 40(2), 139-57. https://doi.org/10.1086/447369

Murillo, F. J. y Román, M. (2008). Resultados de aprendizaje en América Latina a partir de las evaluaciones nacionales. Revista Iberoamericana de Evaluación Educativa, 1(1), 6-35.

National Mathematics Advisory Panel. (2008). Foundations for success: report of the task group on teachers and teacher education. U.S. Department of Education.

oCDE (2016). Estudiantes de bajo rendimiento: por qué se quedan atrás y cómo ayudarles a tener éxito. Autor. http://www.oecd.org/pisa/keyfindings/PISA-2012-Estudiantes-de-bajo-rendimiento.pdf

OCDE (2018). Evaluaciones de políticas nacionales de educación. Educación en Chile. Autor. http://www.oecdilibrary.org/education/educacion-en-chile 9789264288720-es

Pino-Fan, L., Godino, J. D. y Font, V. (2018). Assessing key epistemic features of didactic-mathematical knowledge of prospective teachers: the case of the derivative. Journal of Mathematics Teacher Education, 21(1), 63-94.

Pinto, H. y Ribeiro, C. M. (2013). Conhecimento e formação de futuros professores dos primeiros anos - o sentido de número racional [Conocimiento y formación de futuros profesores de los dos primeros anos el sentido del número racional]. Da Investigação às Práticas, 3(1), 85-105.

Rittle-Johnson, B. y Schneider, M. (2014). Developing conceptual and procedural knowledge of mathematics. En R. Kadosh y A. Dowker (Eds.), Oxford Handbook of Numerical Cognition. Oxford University Press.

Rittle-Johnson, B., Schneider, M. y Star, J. R. (2015). Not a one-way street: bidirectional relations between procedural and conceptual knowledge of mathematics. Educational Psychology Review, 27(4), 587-597. https://doi.org/10.1007/s10648-015-9302-x

Rodríguez, P. de la C. y Olfos, R. (2018). Instrumentos consistentes para la enseñanza de fracciones en 40. grado. Revista Electrónica de Investigación Educativa, 20(1), 48-58.

https://doi.org/10.24320/redie.2018.20.1.1358

Rowland, T., Huckstep, P. y Thwaites, A. (2005). Elementary teachers' mathematics subject knowledge: the knowledge quartet and the case of Naomi. Journal of Mathematics Teacher Education, 8(3), 255-281. https://doi.org/10.1007/s10857-005-0853-5

Shulman, L. S. (1986). Those who understand: Knowledge growth in teaching. Educational Researcher, 15(2), 4-14. http://www.fisica.uniud.it/URDF/masterDidSciUD/materiali/pdf/Shulman 1986.pdf

Siegler, R. S., Duncan, G. J., Davis-Kean, P. E., Duckworth, K., Claessens, A., Engel, M., Susperreguy, M. I. y Chen, M. (2012). Early predictors of high school mathematics achievement. Psychological Science, 23(7), 691-697. https://doi.org/10.1177/0956797612440101 
Stylianides, A. y Stylianides, G. (2014). Viewing "mathematics for teaching" as a form of applied mathematics: implications for the mathematical preparation of teachers. Notices of the AMS, 61(3), 266-276.

Tchoshanov, M. A. (2011). Relationship between teacher knowledge of concepts and connections, teaching practice, and student achievement in middle grades mathematics. Educational Studies in Mathematics, 76(2), 141-164. https://doi.org/10.1007/s10649-010-9269-y

Tchoshanov, M., Cruz, M. D., Huereca, K., Shakirova, K., Shakirova, L. e Ibragimova, E. N. (2017). Examination of lower secondary mathematics teachers' content knowledge and its connection to students' performance. International Journal of Science and Mathematics Education, 15(4), 683-702. https://doi.org/10.1007/s10763-015-9703-9

Torbeyns, J., Schneider, M., Xin, Z. y Siegler, R. (2015). Bridging the gap: fraction understanding is central to mathematics achievement in students from three different continents. Learning and Instruction, 37, 513. https://doi.org/10.1016/i.learninstruc.2014.03.002

Tuñón, I. y Poy, S. (2016). Factores asociados a las calificaciones escolares como proxy del rendimiento educativo. Revista Electrónica de Investigación Educativa, 18(1), 98-111.

http://redie.uabc.mx/redie/article/view/615 